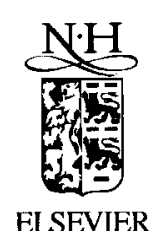

Comput. Methods Appl. Mech. Engrg. 152 (1998) 73-84

\title{
A hierarchical finite element method based on the partition of unity
}

\author{
Robert L. Taylor ${ }^{\mathrm{a}, *, 1}$, O.C. Zienkiewicz ${ }^{\mathrm{b}, 2}$, E. Oñate ${ }^{\mathrm{c}}$ \\ a Department of Civil Engineering, University of California, Berkeley, CA 94720, USA \\ b Department of Civil Engineering, University College, Swansea, UK \\ ' Director CIMNE, Universitat Politécnica de Catalunya, Barcelona, Spain
}

Dedicated to J. Tinsley Oden on the occasion of his 60th birthday

Received 17 January 1997

\begin{abstract}
In this paper we consider the application of hierarchical functions to base approximations which are a partition of unity. The particular hierarchical functions used are added to base finite element interpolations which, for $C^{0}$ approximations, are a particular case of the partition of unity. We also show how the functions may be constructed to preserve the interpolation property of the base finite element functions. An application to linear elasticity is used to illustrate the properties and stability of the approximation.
\end{abstract}

\section{Introduction}

The use of hierarchical interpolations for finite element analyses where approximations must be only $C^{0}$ continuous usually adds functions to the edges, faces and interior of low order isoparametric interpolations (see e.g. [1]). For example, using a simple triangular element with linear interpolations quadratic and higher order functions may be added to each edge in a direct manner. To create complete polynomial approximations of cubic or higher order over the triangle it is necessary to also add an interior term. In recent publications [2-5], it has been shown that hierarchical functions may be added to any base approximation which is a partition of unity [6] and recover globally the base approximation and the added functions. Using this approach it is possible to have polynomial orders which vary from point to point in a mesh. Moreover, the addition of each polynomial term can be associated with nodes of the base approximation, without a need to add different types of functions for edges, faces and/or interior points. Thus, programming complexity for the approach can be significantly reduced.

In this paper we investigate the properties of the new hierarchical functions in the solution of any finite element problem for which $C^{0}$ interpolations may be employed. In Section 2, we show how the functions may be given in a form which does not destroy the original interpolation capability of the base interpolations. The modified form for interpolation is shown to preserve the property of high order global Cartesian polynomial approximation while being straightforward to implement.

\footnotetext{
${ }^{*}$ Corresponding author.

${ }^{1}$ Visiting Professor, Universitat Politécnica de Catalunya

${ }^{2}$ UNESCO Professor, Universitat Politécnica de Catalunya, Barcelona, Spain
} 
In Section 3, we illustrate the basic structure for application of the method to plane strain, linear elasticity problems. Patch test evaluations, as described in $[1,7]$ are used to assess the stability of the procedure using the simplest quadratic elements on triangles and quadrilaterals. Certain forms of the interpolation are found to be stable while others are not. Finally, for the triangular element form the method is applied and compared to classical isoparametric 6-node triangular element solutions.

\section{Hierarchical interpolation}

The basic form of the hierarchical approximation in one dimension is

$$
\phi(x)=\sum_{a=1}^{N} N_{a}^{k}(x)\left[\bar{\phi}_{a}+\boldsymbol{P}^{k}(x) \overline{\boldsymbol{b}}_{a}\right]
$$

with

$$
\boldsymbol{P}^{k}(x)=\left[\begin{array}{lll}
p_{1}(x) & p_{2}(x) & \cdots
\end{array}\right]
$$

and

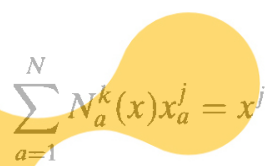

for $j=0,1, \ldots, k$ and any $x$. Note that for $j$ zero the above implies that

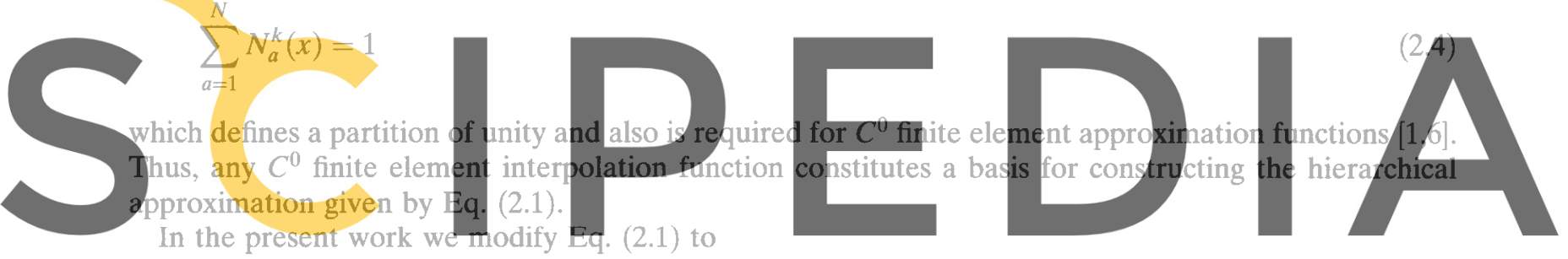

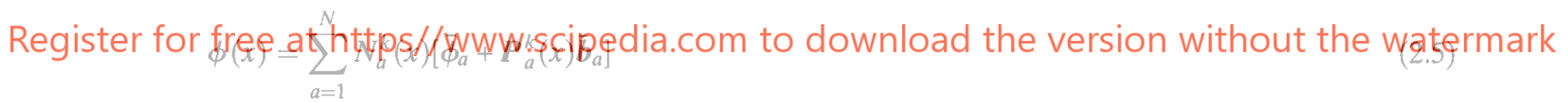

and use polynomial approximation for the $\boldsymbol{P}_{a}^{k}$.

The first derivative of Eq. (2.5) in each element is given by

$$
\frac{\mathrm{d} \phi}{\mathrm{d} x}=\sum_{a=1}^{N}\left\{\frac{\mathrm{d} N_{a}^{k}}{\mathrm{~d} x} \bar{\phi}_{a}+\left[\frac{\mathrm{d} N_{a}^{k}}{\mathrm{~d} x} \boldsymbol{P}_{a}^{k}+N_{a}^{k} \frac{\mathrm{d} P_{a}^{k}}{\mathrm{~d} x}\right] \overline{\boldsymbol{b}}_{a}\right\}
$$

The following hierarchical forms are used for the work reported here:

Form $A$ in which the polynomials are constructed as

$$
\boldsymbol{P}_{a}^{k}(x)=\left[\begin{array}{lll}
\left(x-x_{a}\right)^{k+1} & \left(x-x_{a}\right)^{k+2} & \ldots
\end{array}\right]
$$

or

Form $B$ in which the polynomials are constructed as

$$
\boldsymbol{P}_{a}^{k}(x)=\left[\begin{array}{lll}
\left(x^{k+1}-x_{a}^{k+1}\right) & \left(x^{k+2}-x_{a}^{k+2}\right) & \cdots
\end{array}\right]
$$

Note that the hierarchical functions are also now associated to each node, $a$. 


\subsection{Form A: One dimensional approximations}

To illustrate the performance of the approximation given by Eq. (2.5), we consider a one-dimensional element with two nodes and take

$$
x=\sum_{a=1}^{2} N_{a}^{1}(\xi) \bar{x}_{a}
$$

where $\xi$ is a natural coordinate satisfying

$$
-1 \leqslant \xi \leqslant 1
$$

and $N_{a}^{1}$ are shape functions in each element given by

$$
N_{1}^{1}(\xi)=\frac{1}{2}(1-\xi) ; \quad N_{2}^{1}(\xi)=\frac{1}{2}(1+\xi)
$$

For a quadratic polynomial approximation use the form given by Eq. (2.7) with

$$
\mathbb{P}_{a}^{1}(x)=\left(x-x_{a}\right)^{2}
$$

Thus, within each element the approximation is given by

$$
\phi(x)=\sum_{a=1}^{2} N_{a}^{1}(x)\left[\bar{\phi}_{a}+\left(x-x_{a}\right)^{2} \bar{b}_{a}\right]
$$

Let us now verify that the approximation contains the polynomials
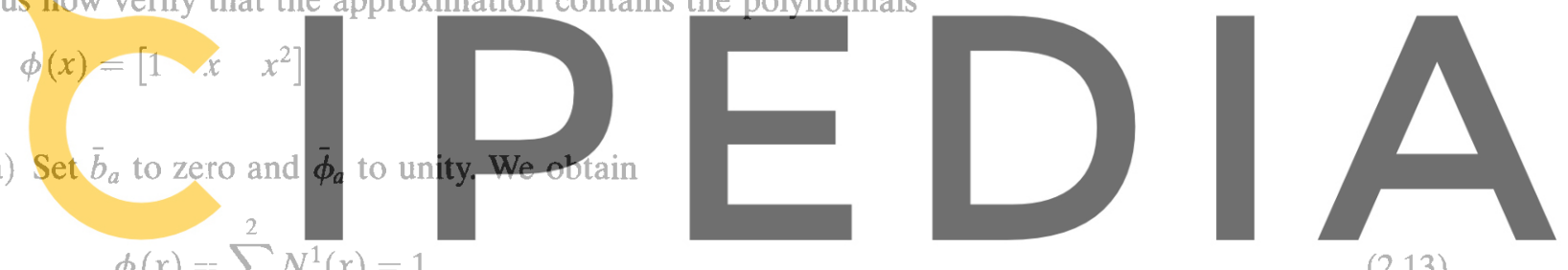

(2.13)

Register for free at https//www.scipedia.com to download the version without the watermark

(b) Set $\bar{b}_{a}$ to zero and $\bar{\phi}_{a}$ to $x_{a}$. We obtain

$$
\phi(x)==\sum_{a=1}^{2} N_{a}^{1}(x) x_{a}=x
$$

(c) Set $\bar{b}_{a}$ to -1 and $\bar{\phi}_{a}$ to $x_{a}^{2}$. We obtain

$$
\phi(x)==\sum_{a=1}^{2} N_{a}^{1}(x)\left[x_{a}^{2}-\left(x-x_{a}\right)^{2}\right]
$$

which expands to

$$
\phi(x)==\sum_{a=1}^{2} N_{a}^{1}(x)\left[2 x x_{a}^{2}-x^{2}\right]
$$

and by Eq. (2.3) becomes

$$
\phi(x)=x^{2}
$$

Joining elements to form an arbitrary patch preserves the above properties for global polynomials. Thus, the polynomial form given by Eqs. (2.12) interpolates through points located at $x_{a}$ and contains complete global polynomials to order 2 . 


\subsection{Form B: One-dimensional approximations}

Form B may also be used to perform the interpolations. Accordingly, for the one-dimensional case with quadratic polynomial approximation we introduce Eq. (2.8) as

$$
\boldsymbol{P}_{a}^{1}(x)=\left(x^{2}-x_{a}^{2}\right)
$$

Thus, within each element the approximation for this form is given by

$$
\phi(x)=\sum_{a=1}^{2} N_{a}^{1}(x)\left[\bar{\phi}_{a}+\left(x^{2}-x_{a}^{2}\right) \bar{b}_{a}\right]
$$

Let us now verify that this approximation contains the polynomials

$$
\phi(x)=\left[\begin{array}{lll}
1 & x & x^{2}
\end{array}\right]
$$

(a) Set $\bar{b}_{a}$ to zero and $\bar{\phi}_{a}$ to unity. We obtain

$$
\phi(x)=\sum_{a=1}^{2} N_{a}^{1}(x)=1
$$

\section{(b) Set $\bar{b}_{a}$ to zero and $\bar{\phi}_{a}$ to $x_{a}$. We obtain}

$$
\phi(x)=\sum_{a=1}^{2} N_{a}^{1}(x) x_{a}=x
$$

(c) Set $\bar{b}_{a}$ to 1 and $\bar{\phi}_{a}$ to $x_{a}^{2}$. We obtain
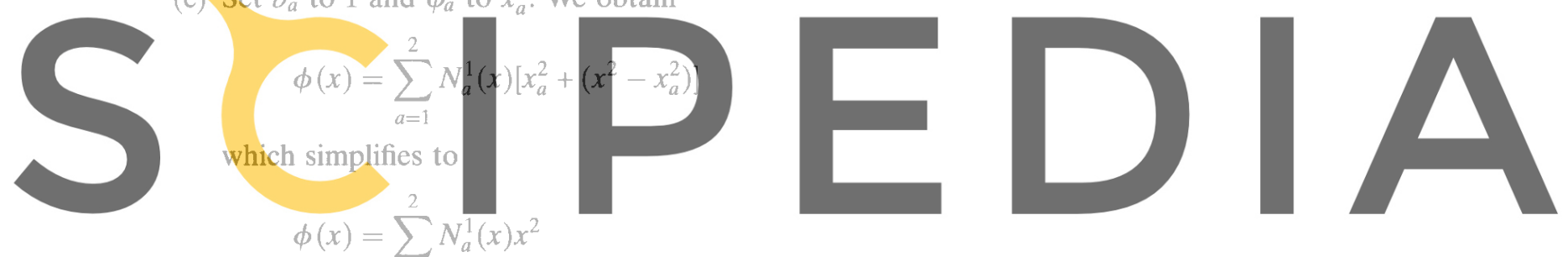

Register for free at http ${ }^{a} \bar{s} / /$ www.scipedia.com to download the version without the watermark and using Eq. (2.3) becomes

$$
\phi(x)=x^{2}
$$

Joining elements to form an arbitrary patch preserves the above properties for global polynomials. Thus, the polynomial form given by Eq. (2.17) also interpolates through points located at $x_{a}$ and contains complete global polynomials to order 2 .

It is evident that the modification given by Eq. (2.5) also makes development of finite elements much simpler than using Eq. (2.1) since the nodal parameters $\bar{\phi}_{a}$ retain their original finite element physical meaning. Furthermore, the specification of essential and natural boundary conditions may directly use the interpolation property.

\subsection{Two-dimensional approximations}

For two-dimensional problems, the approximations in each element may be taken as

$$
\boldsymbol{x}(\boldsymbol{\xi})=\sum_{a=1}^{N} N_{a}^{k}(\boldsymbol{\xi}) \overline{\boldsymbol{x}}_{a}
$$

for coordinates and

$$
\boldsymbol{\phi}(\boldsymbol{\xi})=\sum_{a=1}^{N} N_{a}^{k}(\boldsymbol{\xi})\left[\overline{\boldsymbol{\phi}}_{a}+\boldsymbol{P}_{a}^{k}(\boldsymbol{x}(\boldsymbol{\xi})) \overline{\boldsymbol{b}}_{a}\right]
$$


for dependent variables. In the above $N$ is the number of nodes on each element and the $\boldsymbol{\xi}$ are appropriate natural coordinates.

\subsubsection{Three node triangular element}

The simplest element in two dimensions is a triangle with three nodes where $\xi$ are area coordinates, $\xi_{1}, \xi_{2}, \xi_{3}$ which satisfy

$$
0 \leqslant \xi_{i} \leqslant 1
$$

and

$$
\xi_{1}+\xi_{2}+\xi_{3}=1
$$

The shape functions for the triangle are given as

$$
N_{a}^{1}=\xi_{a}
$$

The hierarchical terms for Form A are similar to Eq. (2.7) and here are taken as

$$
P_{a}^{1}(x(\xi))=\left[\begin{array}{lll}
\left(x-x_{a}\right)^{2} & \left(x-x_{a}\right)\left(y-y_{a}\right) & \left(y-y_{a}\right)^{2}
\end{array}\right]
$$

with parameters

$$
\overline{\boldsymbol{b}}_{a}=\left[\begin{array}{l}
\bar{b}_{a}^{x x} \\
\bar{b}_{a}^{x y} \\
\bar{b}_{a}^{y y}
\end{array}\right]
$$

The approximation for Form B becomes

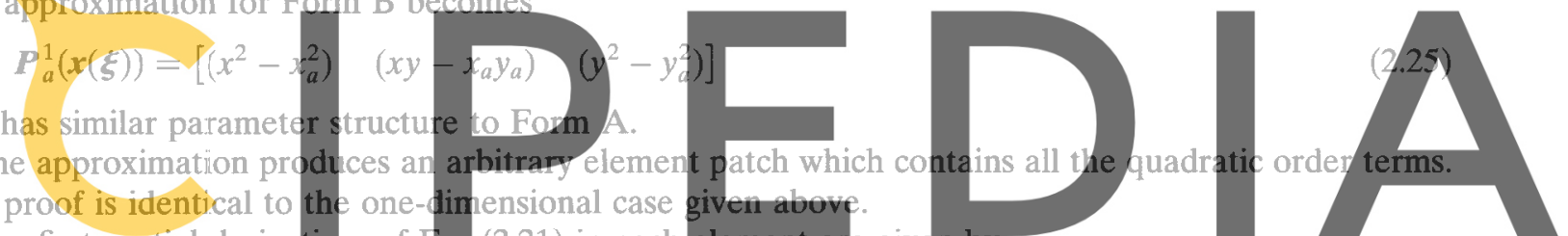

The first partial derivatives of Eq. (2.21) in each element are given by

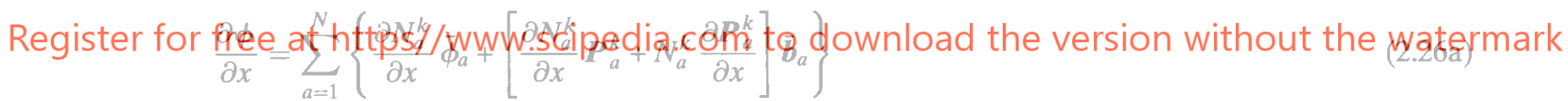

and

$$
\frac{\partial \phi}{\partial y}=\sum_{a=1}^{N}\left\{\frac{\partial N_{a}^{k}}{\partial y} \bar{\phi}_{a}+\left[\frac{\partial N_{a}^{k}}{\partial y} \boldsymbol{P}_{a}^{k}+N_{a}^{k} \frac{\partial \boldsymbol{P}_{a}^{k}}{\partial y}\right] \overline{\boldsymbol{b}}_{a}\right\}
$$

For the quadratic polynomial case we have, for Form A, the result

$$
\frac{\partial \boldsymbol{P}_{a}^{k}}{\partial x}=\left[\begin{array}{lll}
2\left(x-x_{a}\right) & \left(y-y_{a}\right) & 0
\end{array}\right]
$$

and

$$
\frac{\partial \boldsymbol{P}_{a}^{k}}{\partial y}=\left[\begin{array}{lll}
0 & \left(x-x_{a}\right) & 2\left(y-y_{a}\right)
\end{array}\right]
$$

whereas for Form $B$ the result is

$$
\frac{\partial \boldsymbol{P}_{a}^{k}}{\partial x}=\left[\begin{array}{lll}
2 x & y & 0
\end{array}\right]
$$

and

$$
\frac{\partial \boldsymbol{P}_{a}^{k}}{\partial y}=\left[\begin{array}{lll}
0 & x & 2 y
\end{array}\right]
$$


For problems in which the coordinates attain large values, Form A leads to better numerical conditioning in the evaluation of integrals. For the quadratic interpolation case, we have for Form $B$ the result

$$
\boldsymbol{\phi}(\boldsymbol{\xi})=\sum_{a=1}^{N} N_{a}^{k}(\boldsymbol{\xi})\left[\hat{\boldsymbol{\phi}}_{a}+\left[x^{2}, x y, y^{2}\right] \overline{\boldsymbol{b}}_{a}\right]
$$

where

$$
\hat{\phi}_{a}=\bar{\phi}_{a}-x_{a}^{2} \bar{b}_{a}^{x x}-x_{a} y_{a} \bar{b}_{a}^{x y}-y_{a}^{2} \bar{b}_{a}^{y y}
$$

Expansion of Form A produces results which include additional terms involving the linear polynomials $x$ and $y$. Hence, Form B merely changes the definition of the parameters defining the original finite element interpolations whereas Form A changes the structure of the original interpolation. Both forms, however, are legitimate quadratic hierarchical interpolations.

Other interpolation types and orders may be obtained by changing the dimension of the problem, the order of the basic approximations $N_{a}^{k}$, and/or the order of the terms included in $\boldsymbol{P}_{a}^{k}$. For example, if the basic approximations are $N_{a}^{2}$ (i.e. contain all polynomials up to order 2) a cubic interpolation for Form A may be constructed using Eq. (2.5) with

$$
\boldsymbol{P}_{a}^{2}(x)=\left[\begin{array}{llll}
\left(x-x_{a}\right)^{3} & \left(x-x_{a}\right)^{2}\left(y-y_{a}\right) & \left(x-x_{a}\right)\left(y-y_{a}\right)^{2} & \left(y-y_{a}\right)^{3}
\end{array}\right]
$$

Similar constructions may also be performed for two-dimensional quadrilateral elements or threedimensional tetrahedra or brick elements. As noted in [8], however, we must ensure that no linear dependencies occur in the final interpolation form.

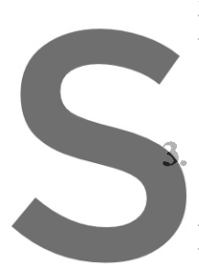

It is possible to introduce other types of function
Application to linear elasticity
In the previous section the form for polynomial in
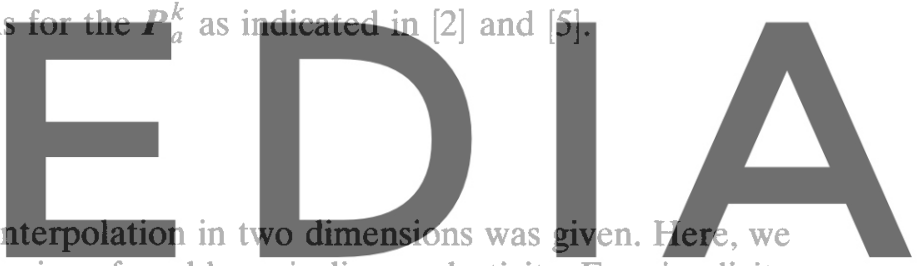

consider the use of the interpolation to model behavior of problems in linear elasticity. For simplicity

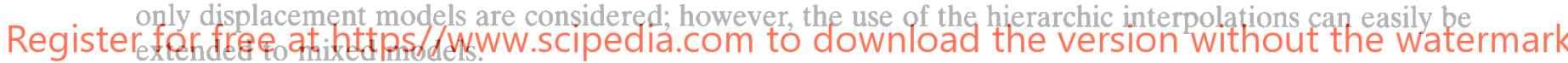

For a displacement model the finite element arrays may be deduced from the principal of minimum potential energy given here by

$$
\Pi(\boldsymbol{u})=\int_{\Omega} W(\boldsymbol{\epsilon}) \mathrm{d} \Omega-\int_{\Omega} \boldsymbol{u}^{\mathrm{T}} \boldsymbol{b} \mathrm{d} \Omega-\int_{\Gamma_{i}} \boldsymbol{u}^{\mathrm{T}} \overline{\boldsymbol{t}} \mathrm{d} \Omega
$$

where $\boldsymbol{u}$ are displacement components, $\boldsymbol{\epsilon}$ are strains, $W$ the strain energy, $\boldsymbol{b}$ body forces, and $\overline{\boldsymbol{t}}$ specified boundary tractions. The strain-displacement relations are given by

$$
\boldsymbol{\epsilon}=\nabla^{(\mathrm{s})} \boldsymbol{u}
$$

where $\nabla^{(\mathrm{s})}$ denotes the symmetric part of the displacement gradient. For two-dimensional plane strain problems, the strain-displacement relations may be written in matrix form as

$$
\boldsymbol{\epsilon}=\left[\begin{array}{c}
\frac{\partial u}{\partial x} \\
\frac{\partial v}{\partial y} \\
\frac{\partial u}{\partial y}+\frac{\partial v}{\partial x}
\end{array}\right]
$$

Inserting the interpolations for $u$ and $v$ given by Eq. (2.5), the strain-displacement relations become 


$$
\boldsymbol{\epsilon}=\sum_{a-1}^{N}\left[\begin{array}{cc}
\frac{\partial N_{a}^{k}}{\partial x} & 0 \\
0 & \frac{\partial N_{a}^{k}}{\partial y} \\
\frac{\partial N_{a}^{k}}{\partial y} & \frac{\partial N_{a}^{k}}{\partial x}
\end{array}\right]\left[\begin{array}{c}
\bar{u}_{a} \\
\bar{v}_{a}
\end{array}\right]+\sum_{a=1}^{N}\left[\begin{array}{cc}
\left(\frac{\partial N_{a}^{k}}{\partial x} \boldsymbol{P}_{a}^{k}+N_{a}^{k} \frac{\partial \boldsymbol{P}_{a}^{k}}{\partial x}\right) & \left(\frac{\partial N_{a}^{k}}{\partial x} \boldsymbol{P}_{a}^{k}+N_{a}^{k} \frac{\partial \boldsymbol{P}_{a}^{k}}{\partial y}\right) \\
0 & \left(\frac{\partial N_{a}^{k}}{\partial x} \boldsymbol{P}_{a}^{k}+N_{a}^{k} \frac{\partial \boldsymbol{P}_{a}^{k}}{\partial x}\right)
\end{array}\right]\left[\begin{array}{c}
\overline{\boldsymbol{b}}_{a}^{u} \\
\overline{\boldsymbol{b}}_{a}^{v}
\end{array}\right]
$$

The first term is identical to usual finite element strain-displacement matrices (see e.g. [1]) and the second term has identical structure to the usual arrays. Thus, the development of all element arrays follows standard procedures.

\subsection{Quadratic triangular element}

For a triangular element with linear interpolation the shape functions and quadratic polynomial hierarchic terms are given by Eqs. (2.22) and (2.23) or (2.25), respectively. Using isoparametric concepts the coordinates are given by

$$
\boldsymbol{x}=\sum_{a=1}^{3} N_{a}^{1} \overline{\boldsymbol{x}}_{a}=\sum_{a=1}^{3} \xi_{a} \overline{\boldsymbol{x}}_{a}
$$

and are used to construct all polynomials appearing in the hierarchical form.

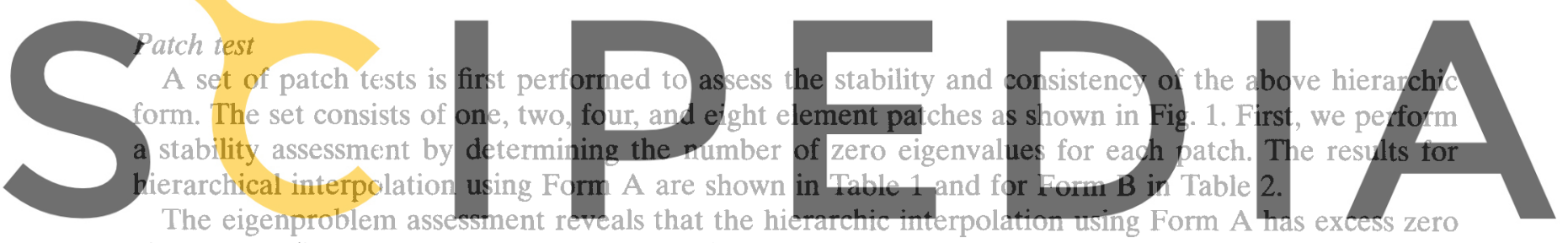

The eigenproblem assessment reveals that the hierarchic interpolation using Form A has extess zero

eigenvalues (i.e. spurious zero energy modes) only for meshes consisting of one or two elements. Fur-

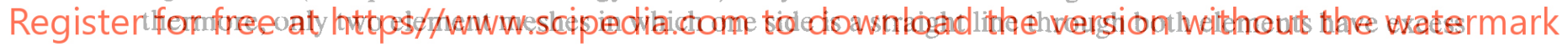

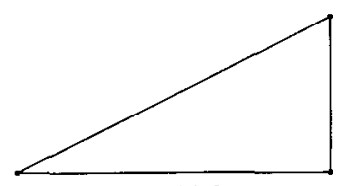

(a) One element

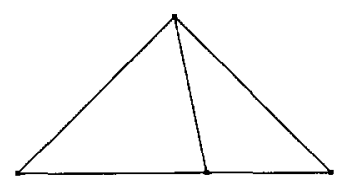

(c) Two element, b

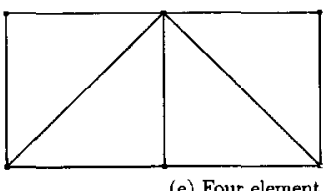

(c) Four element

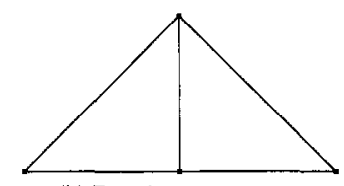

(b) Two element, a

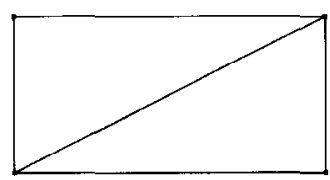

(d) Two element,

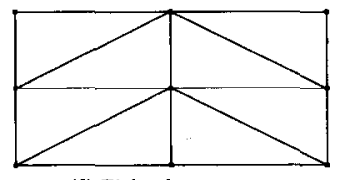

(f) Eight element

Fig. 1. Patches for eigenproblem assessment. 
Table 1

Triangle patch tests for Form A: Number zero eigenvalues, minimum non-zero value, and maximum value

\begin{tabular}{llll}
\hline Mesh & No. zero & Min. value & Max. value \\
\hline 1 & 7 & $4.7340 \mathrm{E}+01$ & $2.0560 \mathrm{E}+06$ \\
$2 \mathrm{a}$ & 5 & $4.0689 \mathrm{E}+01$ & $2.1543 \mathrm{E}+05$ \\
$2 \mathrm{~b}$ & 5 & $4.1971 \mathrm{E}+02$ & $2.2648 \mathrm{E}+05$ \\
$2 \mathrm{c}$ & 3 & $1.5728 \mathrm{E}+02$ & $2.3883 \mathrm{E}+06$ \\
4 & 3 & $1.0446 \mathrm{E}+02$ & $2.9027 \mathrm{~F}+05$ \\
8 & 3 & $9.5560 \mathrm{E}+01$ & $3.4813 \mathrm{E}+05$ \\
\hline
\end{tabular}

Table 2

Triangle patch tests for Form B: Number zero eigenvalues, minimum non-zero value, and maximum value

\begin{tabular}{llll}
\hline Mesh & No. zero & Min. value & Max. value \\
\hline 1 & 7 & $4.6067 \mathrm{E}+01$ & $1.0120 \mathrm{E}+07$ \\
$2 \mathrm{a}$ & 7 & $4.5559 \mathrm{E}+01$ & $1.8880 \mathrm{E}+06$ \\
$2 \mathrm{~b}$ & 7 & $4.3815 \mathrm{E}+01$ & $2.2254 \mathrm{E}+06$ \\
$2 \mathrm{c}$ & 7 & $1.6340 \mathrm{E}+02$ & $1.0690 \mathrm{E}+07$ \\
4 & 7 & $1.0847 \mathrm{E}+02$ & $3.7646 \mathrm{E}+06$ \\
8 & 7 & $8.8303 \mathrm{E}+01$ & $5.3064 \mathrm{E}+06$ \\
\hline
\end{tabular}

zero values. Once the mesh has no straight intersections the number of zero modes becomes correct

(e.g. contain only the three rigid body modes). On the other hand, using Form B spurious zero modes persist for all meshes. We note that Form B involves only a simple change of the definition for the finite

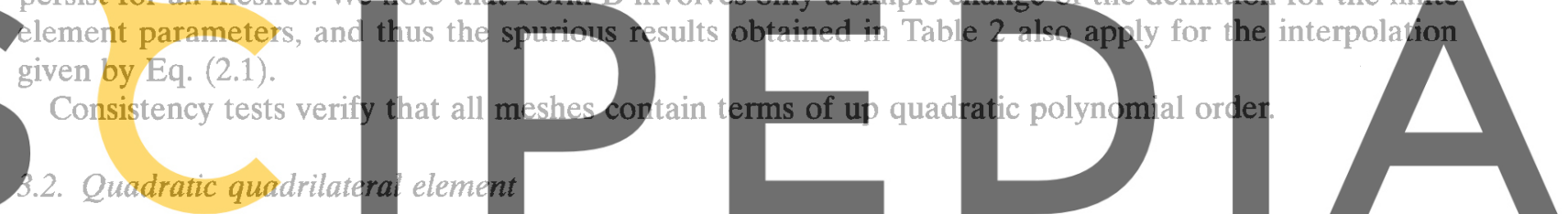

For a quadrilateral element with bi-linear interpolation the shape functions given by

Register for free at https//www.scipedia.com to download the version without the watermark

$$
N_{a}^{1}(\xi, \eta)=\frac{1}{4}\left(1+\xi_{a} \xi\right)\left(1+\eta_{a} \eta\right)
$$

where $\xi, \eta$ are natural coordinates satisfying

$$
-1 \leqslant \xi, \eta \leqslant 1
$$

and $\xi_{a}, \eta_{a}$ are the values of the natural coordinates at vertex nodes. Using isoparametric interpolations the coordinates may be computed from

$$
\boldsymbol{x}=\sum_{u=1}^{4} N_{a}^{1}(\xi, \eta) \boldsymbol{x}_{a}
$$

Quadratic polynomial hierarchic terms are constructed for Form A using Eq. (2.23).

\subsubsection{Patch test}

A set of patch tests is first performed to assess the stability and consistency of the above hierarchic form on rectangles. The set consists of one, two and four element patches as shown in Fig. 2. First, we perform a stability assessment by determining the number of zero eigenvalues for each patch. The results are shown in Table 3.

The eigenproblem assessment reveals that the hierarchic interpolation using Form $\mathrm{A}$ has excess zero eigenvalues (i.e. spurious zero energy modes) for all meshes considered. Further considerations reveal that the use of quadratic polynomials for $P$ which include $x y$ introduce linearly dependent terms since 


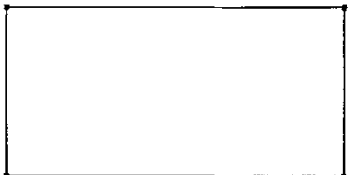

(a) $1 \times 1$ mesh

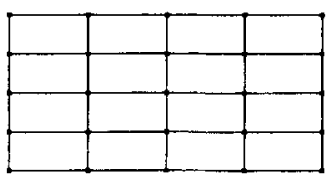

(c) $4 \times 4$ mesh

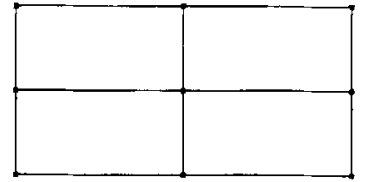

(b) $2 \times 2$ mesh

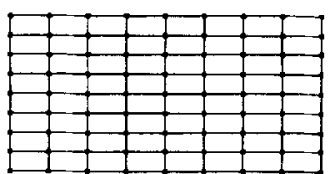

(d) $8 \times 8$ mesh

Fig. 2. Patches for rectangle eigenproblem assessment.

this term is already available when the elements have constant jacobian. Repeating the stability test after eliminating this polynomial yields the results given in Table 4.

Repeating the above for Form B yields the results in Tables 5 and 6 . The eigenproblem assessment reveals that the hierarchic interpolation using Form $B$ also has excess zero eigenvalues for all meshes considered; however, eliminating the polynomial $x y$ again restores proper rank as shown in Table 6.

Consistency tests again confirm the existence of all polynomial terms up to quadratic order.
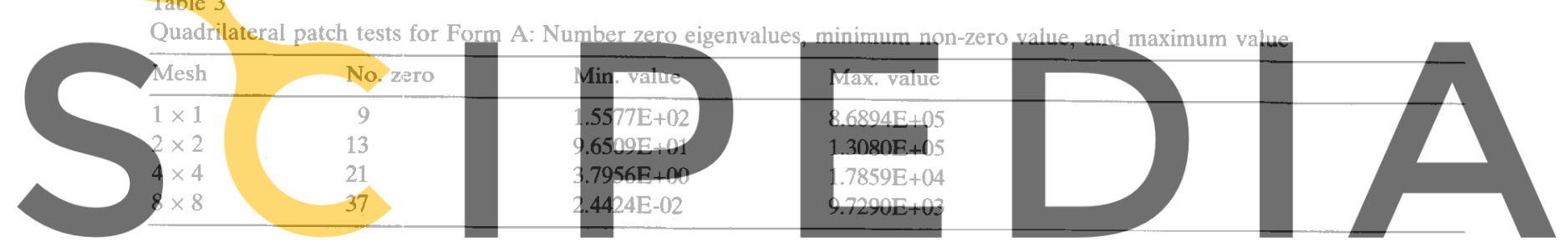

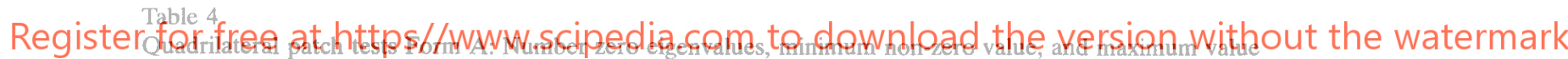

\begin{tabular}{llll}
\hline Mesh & No. zero & Min. value & Max, value \\
\hline $1 \times 1$ & 3 & $1.5739 \mathrm{E}+02$ & $8.6894 \mathrm{E}+05$ \\
$2 \times 2$ & 3 & $9.5719 \mathrm{E}+01$ & $1.3073 \mathrm{E}+05$ \\
$4 \times 4$ & 3 & $4.8047 \mathrm{E}+01$ & $1.7859 \mathrm{E}+04$ \\
$8 \times 8$ & 3 & $4.3963 \mathrm{E}+00$ & $9.7290 \mathrm{E}+03$ \\
\hline
\end{tabular}

Table 5

Quadrilateral patch tests for Form B: Number zero eigenvalues, minimum non-zero value, and maximum value

\begin{tabular}{llll}
\hline Mesh & No. zero & Min. value & Max, value \\
\hline $1 \times 1$ & 9 & $1.5498 \mathrm{E}+02$ & $4.8936 \mathrm{E}+06$ \\
$2 \times 2$ & 9 & $4.8788 \mathrm{E}+01$ & $3.3575 \mathrm{E}+06$ \\
$4 \times 4$ & 9 & $4.4192 \mathrm{E}-01$ & $1.5102 \mathrm{E}+06$ \\
$8 \times 8$ & 9 & $3.0824 \mathrm{E}-03$ & $5.2649 \mathrm{E}+05$ \\
\hline
\end{tabular}

Table 6

Quadrilateral patch tests for Form B: Number zero eigenvalues, minimum non-zero value, and maximum value

\begin{tabular}{llll}
\hline Mesh & No. zero & Min. value & Max. value \\
\hline $1 \times 1$ & 3 & $1.5599 \mathrm{E}+02$ & $4.4176 \mathrm{E}+06$ \\
$2 \times 2$ & 3 & $8.7079 \mathrm{E}+01$ & $3.2108 \mathrm{E}+06$ \\
$4 \times 4$ & 3 & $8.1631 \mathrm{E}+00$ & $9.0948 \mathrm{E}+05$ \\
$8 \times 8$ & 3 & $4.8673 \mathrm{E}-01$ & $4.1174 \mathrm{E}+05$ \\
\hline
\end{tabular}




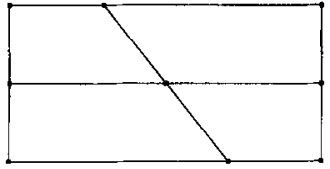

(a) $2 \times 2$ mesh

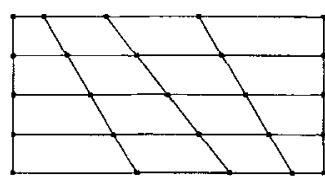

(b) $4 \times 4$ mesh

Fig. 3. Patches for quadrilateral eigenproblem assessment.

The mesh for rectangles was distorted as shown in Fig. 3. With the quadratic polynomial term $x y$ retained, the spurrious eigenvalues persist, and with it removed the consistency test is failed. Thus, the quadrilateral element does not satisfy patch requirements, and in the present work is not considered further.

\subsection{Tension strip}

A finite width strip containing a circular hole with diameter half the width of the strip is subjected to axial extension in the vertical direction. Due to symmetry of the loading and mesh, only one quadrant is discretized. A set of meshes is shown in Figs. 4 and 5. Meshes in Fig. 4 use Form A of the hierarchical interpolation considered above; whereas those in Fig. 5 use standard 6-node isoprarametric quadratic triangles with two degrees of freedom per node (i.e. $u$ and $v$ ).

The problem size and solution statistics are shown in Table 7 for the hierarchical Form A method and in Table 8 for the 6-node isoparametric formulation where mid-side element nodes are permitted to lie on the curved boundary. In Table 9, we present the results when the mid-nodes are linearly intepolated from the vertex nodes (which produces the same boundary interpolation as the new hierarchical form).
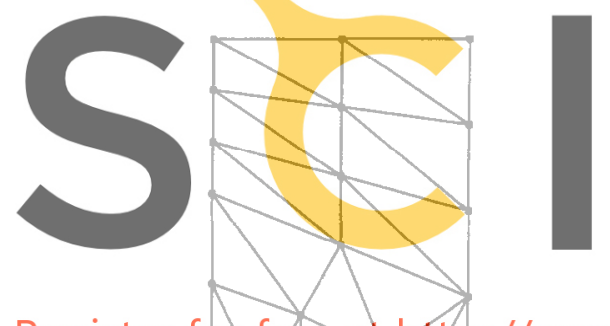

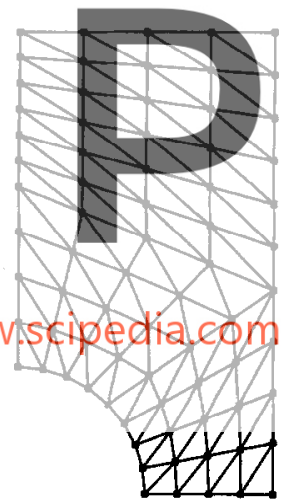

(b) 112 Elements

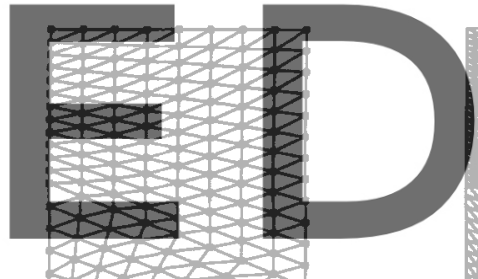

to d ad the version without the watermark

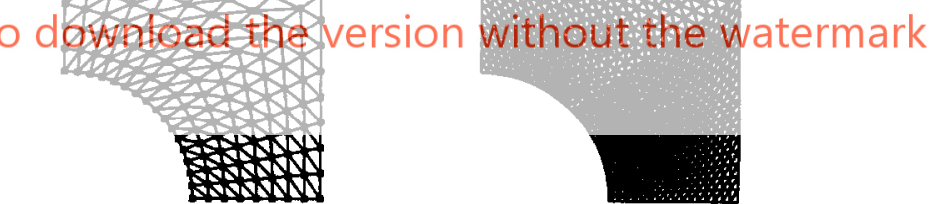

(c) 448 Elements

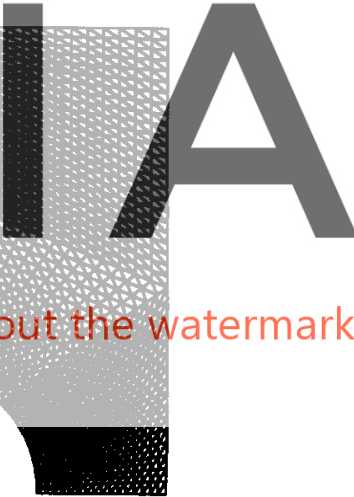

(d) 1792 Elements

Fig. 4 Hierarchic elements form A: Tension strip.

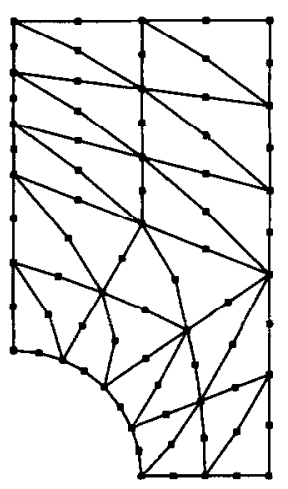

(a) 28 Elements

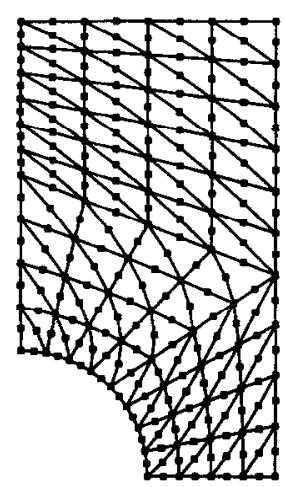

(b) 112 Elements

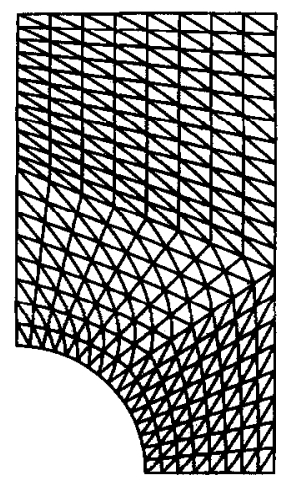

(c) 448 Elements

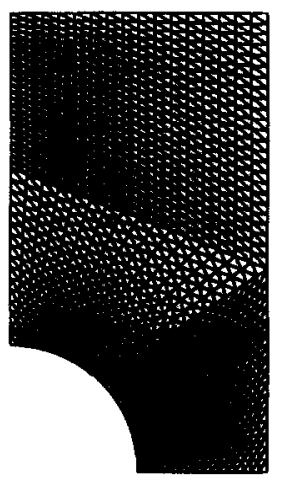

(d) 1792 Elements

Fig. 5. Isoparametric elements: Tension strip. 
Table 7

Hierarchical element problem size statistics

\begin{tabular}{rcccrrr}
\hline Nodes & Elemerits & Equations & Non-zeros & Profile & Column & Energy \\
\hline 30 & 28 & 156 & 2892 & 4159 & 28 & 131.7088 \\
85 & 112 & 537 & 12015 & 29860 & 56 & 127.8260 \\
279 & 448 & 1971 & 48741 & 207508 & 106 & 126.7641 \\
1003 & 1792 & 7527 & 196113 & 1547658 & 205 & 126.5908 \\
\hline
\end{tabular}

Table 8

Isoparametric element problem size statistics. Boundary segments have curved sides

\begin{tabular}{rcccrrr}
\hline Nodes & Elements & Equations & Non-zeros & Profile & Column & Energy \\
\hline 30 & 28 & 129 & 1070 & 1777 & 14 & 127.3350 \\
279 & 112 & 483 & 4613 & 13428 & 28 & 126.6483 \\
1003 & 448 & 1863 & 19091 & 100970 & 55 & 126.5661 \\
3795 & 1792 & 7311 & 77615 & 769110 & 106 & 126.5593 \\
\hline
\end{tabular}

Table 9

Isoparametric element problem size statistics. Boundary segments have straight sides

\begin{tabular}{rcccrrr}
\hline Nodes & Elements & Equations & Non-zeros & Profile & Column & Energy \\
\hline 30 & 28 & 129 & 1070 & 1777 & 14 & 128.6458 \\
279 & 112 & 483 & 4613 & 13428 & 28 & 126.9451 \\
1003 & 448 & 1863 & 19091 & 100970 & 55 & 126.6090 \\
3795 & 1792 & 7311 & 77615 & 769110 & 106 & 126.5394 \\
\hline
\end{tabular}

Table 10

Linear triangular element problem size statistics

\begin{tabular}{rrrrrrr}
\hline Nodes & Elements & Equations & Non-zeros & Profile & Column & Energy \\
\hline 30 & 28 & 36 & 150 & 252 & 7 & 137.652 \\
85 & 112 & 129 & 669 & 1711 & 13 & 131.065 \\
279 & 448 & 483 & 2799 & 12438 & 26 & 128.008 \\
1003 & 1792 & 1863 & 11427 & 94696 & 50 & 126.958 \\
3795 & 7168 & 7311 & 46155 & 738972 & 102 & 126.662 \\
\hline
\end{tabular}

Finally, in Table 10 we present the results using 3-node linear trianglar elements. For the analyses, the material is taken as linear elastic with $E=1000$ and $\nu=0.25$. The half width of the strip is 10 units and the half height is 18 units. The hole has radius 5 .

The best results obtained are consistently for the 6-node isoparametric element. The effect of boundary error is negligible for this problem, especially for the finer meshes. The new element, however, is consistently better than the 3-node triangular element. Consequently, the potential of the new interpolation for adaptive refinement should be evaluated.

\section{Closure}

In this work we have discussed the use of hierarchical approximations which are appended to convention $C^{0}$ finite element interpolations. The form is based on adding polynomial functions to a partition of unity. The particular form considered permits polynomials to any degree to be connected around any node in a mesh. Thus, the method is particularly amenable to adaptive processes. Furthermore, we have shown how the polynomials may be constructed to preserve the interpolating property of the original finite element based functions. For linear elasticity problems, as well as for any form requiring $C^{0}$ approximations, the interpolation preserves the structure of basic finite element strain-displacement matrices and, thus, permits a simple construction of basic arrays.

Based on patch test assessements for the linear elasticity problem, we have demostrated that some forms are stable, while others are not. In particular, triangles based on Form $\mathrm{A}$ achieve stability provided 
more than two elements are used; whereas, Form B interpolation never achieved stability for all boundary conditions. The case of rectangular elements achieved stability provided the interpolation was constructed to avoid linear dependencies. However, the results for quadrilateral elements indicated that neither Form A nor Form B could produce interpolations which were both stable and consistent to quadratic polynomial order. Hence, further study is necessary to achieve success for this class of elements.

Finally, we have compared the formulation for meshes composed of elements with quadratic accuracy at all nodes to conventional 6-node isoparametric triangles which for straight sides also achieve quadratic accuracy. Here, results favor the 6-node quadratic triangles: both for efficiency in overall solution effort, as well as for accuracy based on energy. When compared to 3-node triangular elements the new interpolation shows considerable improvement in accuracy; consequently, further tests in an adaptive environment are needed before a more complete assessment may be reached.

\section{References}

[1] O.C. Zienkiewicz and R.L. Taylor, The Finite Element Method, 4th edition (McGraw Hill; Vol. I 1989, Vol. II 1991).

[2] I. Babuška and J.M. Melenk, The partition of unity finite element method, Technical Note BN-1185, Institute for Physical Science and Technology, University of Maryland, April 1995.

[3] C.A. Duarte, A review of some meshless methods to solve partial differential equations, TICAM Report 95-06, The University of Texas, May 1995.

[4] C.A. Duarte and J.T. Oden, $H p$ clouds-A meshless method to solve boundary value problems, TICAM Report 95-05, The University of Texas, May 1995.

[5] R.L. Taylor, O. C. Zienkiewicz, E. Oñate and S. Idelsohn, Moving least square approximations for solution of differential equations, CIMNE Report 92, International Center for Numerical Methods in Engineering, Barcelona, Spain, December 1995.

[6] W. Rudin, Principles of Mathematical Analysis 3rd. edition (McGraw Hill, 1976).

[7] O.C. Zienkiewicz and R.L. Taylor, The patch test revisited, CIMNE Report No. 104, International Center for Numerical Methods in Engineering, Barcelona, Spain, December 1996.

[8] J.T. Oden, C.A. Duarte and O.C. Zienkiewicz, A new cloud-based $H p$ finite element method, TICAM Report 96-55, November 1996. 